\title{
Optical resonator with steep internal dispersion
}

\author{
G. Müller, ${ }^{1, *}$ M. Müller, ${ }^{1}$ A. Wicht, ${ }^{1}$ R.-H. Rinkleff, ${ }^{1}$ and K. Danzmann ${ }^{1,2}$ \\ ${ }^{1}$ Institut für Atom- und Molekülphysik, Universität Hannover, Callinstraße 38, D-30167 Hannover, Germany \\ ${ }^{2}$ Max-Planck-Institut für Quantenoptik, Außenstelle Hannover, Callinstraße 38, D-30167 Hannover, Germany
}

(Received 2 April 1997)

\begin{abstract}
We present an optical resonator with modified properties due to a nonabsorbing highly dispersive medium. The steep nonabsorbing dispersion is created with an additional pump field in an atomic beam using the effect of coherent population trapping. The linewidth of such a resonator depends on the slope of the dispersion line, which in turn depends on the atomic density and the intensity of pump and probe field. In the experiments presented here, the cavity linewidth is reduced by a factor of more than 50 relative to the linewidth of the empty resonator. We have studied the influence of the relative intensities of pump and probe field on the line profile. Due to the dispersion of the medium, the resonance frequency is nearly independent of the geometrical length of the resonator. [S1050-2947(97)10709-0]
\end{abstract}

PACS number(s): 42.50.Gy, 42.60.Da, 07.60.Ly, 42.62.Fi

\section{INTRODUCTION}

The phenomenon of coherent population trapping (CPT) in media that are interacting with two electromagnetic waves has stimulated a lot of theoretical and experimental interest. A very good overview of the experimental and theoretical results and discussed applications was written by Arimondo [1]. A mechanical analogy found by Hemmer and Prentiss [2] is very useful as first steps to qualitatively understanding many of the effects related to CPT.

One of the main attributes of CPT is the cancellation of absorption at the two-photon resonance frequency. It was this property that led to discussions of lasing without inversion [3], which has just been demonstrated experimentally $[4,5]$. For this application the behavior of coherently prepared atoms in an optical resonator is obviously of great interest.

Applications of CPT based on measuring the two-photon resonance frequency have also been discussed. For example, with a proper choice of levels the two-photon resonance frequency could be shifted by an external magnetic field. This effect is used in the magnetometer [6]. The two-photon resonance without external fields is discussed as a frequency standard [7]. For both applications the sensitivity depends on the sensitivity of the spectroscopic technique used. It is well known that the sensitivity of intracavity spectroscopic techniques [8] is often higher than the sensitivity of other techniques.

It is of general interest that the properties of interferometric devices like optical resonators can be manipulated using nonabsorbing dispersive media [9]. This is important, e.g., for the interferometric gravitational wave detectors that are currently planned or under construction (LIGO [10], VIRGO [11], GEO 600 [12], and TAMA [13]). Most techniques used to increase their sensitivity (e.g., power recycling, signal recycling $[14,15])$ involve Fabry-Pérot cavities, and it is desir-

\footnotetext{
*Present address: Institute for Laser Science, University of Electro-Communications, 1-5-1 Chofugaoka Chofu, Tokyo 182, Japan.
}

able to optimize the performance of these cavities.

For all of these applications the transfer functions of an optical resonator including a coherently prepared medium are of great interest. In this work, we present experimental and numerical results concerning the properties of a resonator that contains a coherently prepared medium with nonabsorbing steep dispersion.

\section{THEORY}

To introduce the main idea we will start with a short theory of an optical resonator with steep dispersion inside. The transmitted power $I_{t}$ of an optical resonator of length $L$ is described by the well-known Airy function:

$$
I_{t}=T_{1} T_{2} \frac{1}{1+R_{1} R_{2} e^{-\alpha L}-2 \sqrt{R_{1} R_{2}} e^{-\alpha L / 2} \cos \Phi}
$$

$R_{1}, R_{2}$ are the reflectivities and $T_{1}, T_{2}$ the transmissivities of the mirrors; $\alpha$ is the round-trip absorption coefficient including all losses except the transmission of the mirrors. $\Phi$ is the round-trip phase shift. If $\alpha$ does not depend on the laser frequency, the transmission depends on the round-trip phase shift

$$
N 2 \pi+\Phi=\left(\omega_{0}+\delta \omega\right) \frac{L}{c}
$$

where $\delta \omega$ is the detuning of the laser frequency from the resonance frequency $\omega_{0}$ of the resonator $\left(\omega_{0} L / c=N 2 \pi\right)$. The linewidth [half width at half maximum (HWHM)] in phase space $\Phi_{1 / 2}$ depends on the reflectivities of the mirrors and can easily be calculated from Eq. (1):

$$
\Phi_{1 / 2}=\arccos \left(\frac{1+R_{1} R_{2}-4 \sqrt{R_{1} R_{2}}}{2 \sqrt{R_{1} R_{2}}}\right) .
$$

Now we include in the resonator a medium of length $l$ with an index of refraction $n$ that depends linearly on the laser frequency: 


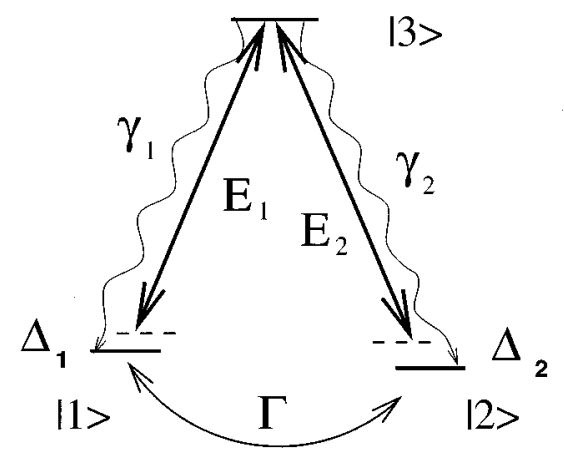

FIG. 1. $\Lambda$ system. The field $E_{1}$ couples the levels $|1\rangle \leftrightarrow|3\rangle$ and the field $E_{2}$ the levels $|2\rangle \leftrightarrow|3\rangle$. The frequency of the laser field $E_{1}$ $\left(E_{2}\right)$ is detuned by $\Delta_{1}\left(\Delta_{2}\right)$ from the atomic resonance frequency. The upper level $|3\rangle$ decays with the rate $\gamma_{1}$ and $\gamma_{2}$ into the levels $|1\rangle$ and $|2\rangle$, respectively, while the coherence between both lower levels decays with $\Gamma$.

$$
n(\omega)=1+\frac{D}{\omega_{0}} \delta \omega
$$

with

$$
D:=\omega_{0} \frac{d n}{\left.d \omega\right|_{\omega=\omega_{0}}} .
$$

Taking only the first-order terms in $\delta \omega$, the round-trip phase shift becomes

$$
\Phi=\delta \omega \frac{L}{c}\left(1+D \frac{l}{L}\right)
$$

i.e., it is amplified by the factor $(1+D l / L)$. Therefore the linewidth of the resonator in frequency space $\delta \omega_{1 / 2}$ (HWHM) is reduced by the same factor:

$$
\delta \omega_{1 / 2}=\frac{c}{L\left(1+D \frac{l}{L}\right)} \arccos \left(\frac{1+R_{1} R_{2}-4 \sqrt{R_{1} R_{2}}}{2 \sqrt{R_{1} R_{2}}}\right) .
$$

If the dispersion is higher than the inverse of the laser frequency $d n / d \omega>\omega^{-1} \approx 3 \times 10^{-15} \mathrm{~s}$, which leads to a $D>1$, the linewidth of the resonator will be reduced. This is one of the possible ways to manipulate the properties of an optical resonator by introducing a dispersive and nonabsorptive medium.

The dispersion of glass $\left(10^{-16}-10^{-18} \mathrm{~s}\right)$ or of other transparent media normally used in optics is orders of magnitude too small to achieve resonators with a smaller bandwidth. The dispersion usually is high enough only near atomic resonances. Unfortunately, here the absorption is much too high, but in several coherently prepared atomic systems narrow nonabsorptive resonances have been found ([1] and references therein), and in the last few years people have been focusing more and more on the dispersive properties of such coherent systems.

A system with steep dispersion at a point of nearly vanishing absorption could be created in a $\Lambda$ system pumped with two coherent laser fields (see Fig. 1). Each field couples
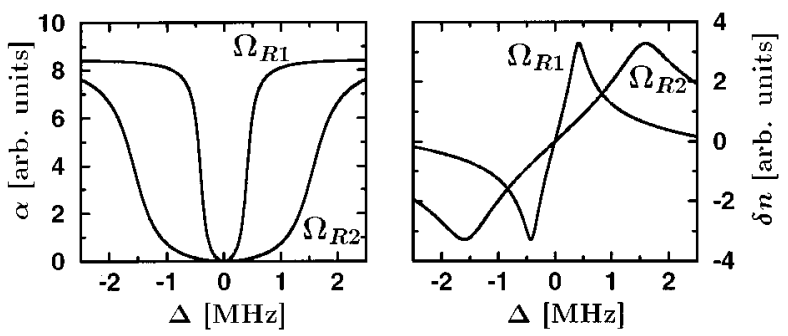

FIG. 2. Absorption $\alpha$ and dispersion $\delta n$ for different effective Rabi frequencies vs detuning $\Delta$. $\Omega_{R 1}=3 \mathrm{MHz}, \Omega_{R 2}=6 \mathrm{MHz} . N$ $=4 \times 10^{9} \mathrm{~cm}^{-3}, \gamma_{1}=\gamma_{2}=5 \mathrm{MHz}, \Gamma=100 \mathrm{~Hz}, \delta v_{\text {Doppler }}=40 \mathrm{MHz}$ (FWHM).

one of the long-lived lower levels $|1\rangle,|2\rangle$ to the upper level $|3\rangle$, with a Rabi frequency $\Omega_{i}=d_{i, 3} E_{i}$ depending on the dipole moments $d_{i, 3}$ of these transitions and on the amplitudes $E_{i}$ of the coupling fields. We will call $E_{1}$ and $E_{2}$ the pump field and the probe field, respectively, but it should be kept in mind that the intensity of the pump field is not necessarily higher than the intensity of the probe field. The upper level could decay to both lower levels with decay rates $\gamma_{1}$ and $\gamma_{2}$. The lower levels thermalize with rate $\Gamma$, normally dominated by the inverse interaction time or the collision rate. The detuning of both fields is given by $\Delta_{1}$ and $\Delta_{2}$, while the detuning from the two-photon resonance is $\Delta=\Delta_{1}-\Delta_{2}$. At the two-photon resonance a destructive interference between the possible interaction channels cancels the absorption. This interference depends on the amplitude and the frequency of both fields.

In such a system the index of refraction for the probe field is (for details see [16], [6], or [17])

$$
n(\Delta)=1+\frac{3}{8 \pi^{2}} \gamma_{2} \lambda^{3} N \frac{\Delta A-\frac{1}{2} \Gamma B}{A^{2}+B^{2}}
$$

while the absorption is

$$
\alpha(\Delta)=\frac{3}{4 \pi} \gamma_{2} \lambda^{2} N \frac{\frac{1}{2} \Gamma A+\Delta B}{A^{2}+B^{2}} .
$$

$N$ is the atomic density and $\lambda$ the wavelength of the laser fields. $A$ and $B$ depend on the effective Rabi frequency $\Omega_{R}$ $=\sqrt{\Omega_{1}^{2}+\Omega_{2}^{2}}$, the decay rates, and also on the detunings:

$$
\begin{gathered}
A=\Omega_{B}^{2}+\frac{1}{4} \Gamma\left(\gamma_{1}+\gamma_{2}\right)-\Delta \Delta_{2}, \\
B=\frac{1}{2}\left[\Delta\left(\gamma_{1}+\gamma_{2}\right)+\Delta_{2} \Gamma\right] .
\end{gathered}
$$

For small detunings $\Delta, \Delta_{2}<\Omega_{R}$, the index of refraction is proportional to the detuning $\Delta$, while the absorption depends on the square of the detuning. In addition, the dispersion and the absorption depend on the effective Rabi frequency and therefore on the sum of the intensities of both fields. In Fig. 2 we have plotted the index of refraction and the absorption for two different Rabi frequencies, taking into account the residual Doppler broadening for our experimental setup, which is in the range of $\delta \nu=40 \mathrm{MHz}$ for the optical transi- 


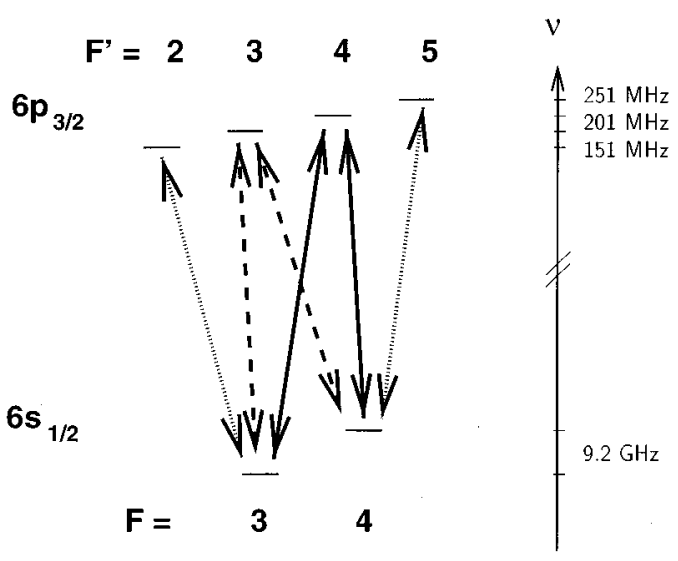

FIG. 3. Atomic levels of cesium used in the experiments. The generation of coherent population trapping between both lower levels $F=3$ and $F=4$ is possible with the upper levels $F^{\prime}=3$ and $F^{\prime}=4$. The upper levels $F^{\prime}=2$ and $F^{\prime}=5$ can only be coupled to one of the lower levels, and therefore these transitions lead even at the two-photon resonance to additional absorption caused by background gas.

tions. For higher Rabi frequencies, which means higher intensities, the dispersion and the absorption are bleached out due to saturation effects.

The first measurements of the index of refraction in a ladder system were made by Xiao et al. [18], and in a $\Lambda$-system by Schmidt et al. [19] and by our group [16]. In our experiments a dispersion of up to $10^{-11} \mathrm{~s}$ at a point of nearly vanishing absorption was measured. This leads to $D$ $\approx 5000$ depending mainly on the atomic density and the laser intensities.

To conclude, a CPT system can generate a nonabsorptive resonance with a steep dispersion. However, for an application inside a resonator, we have to keep in mind that there are now two resonance frequencies, the two-photon resonance of CPT and the resonance frequency of the resonator. These frequencies are usually different.

\section{EXPERIMENT}

In the experiments presented here we used a $\Lambda$ system in the $D_{2}$ line $(852 \mathrm{~nm}$ ) of cesium (see Fig. 3). In this system coherence can be created between the hyperfine levels of the ground state $\left(6 s_{1 / 2} F=3,4\right)$ by coupling both levels to one of the upper levels $6 p_{3 / 2} F^{\prime}=3,4$. One of the problems in our experiments was residual gas in the vacuum chamber. The thermal background gas gave rise to additional absorption due to the other hyperfine levels of the upper state $\left(6 p_{3 / 2}\right.$ $F^{\prime}=2,5$ ), which could only be coupled to one of the lower levels. To reduce this, we used for the pump field the $6 s_{1 / 2}$ $F=4 \leftrightarrow 6 p_{3 / 2} F^{\prime}=4$ transition, and for the probe field the $\left(6 s_{1 / 2} F=3 \leftrightarrow 6 p_{3 / 2} F^{\prime}=4\right)$ transition. With this choice of transitions the absorption of the probe field due to the absorptive transition $6 s_{1 / 2} F=3 \leftrightarrow 6 p_{3 / 2} F^{\prime}=2$ was minimized.

The experimental setup is shown in Fig. 4. The density of the atomic beam in the interaction regions was about 4 $\times 10^{9} \mathrm{~cm}^{-3}$, with an interaction length of $1 \mathrm{~cm}$ in each region. The optical transitions had a residual Doppler broadening of $\delta \nu$ [full width at half maximum (FWHM)] $\approx 40 \mathrm{MHz}$. The laser sources were two laser diodes (LD1, LD2) both

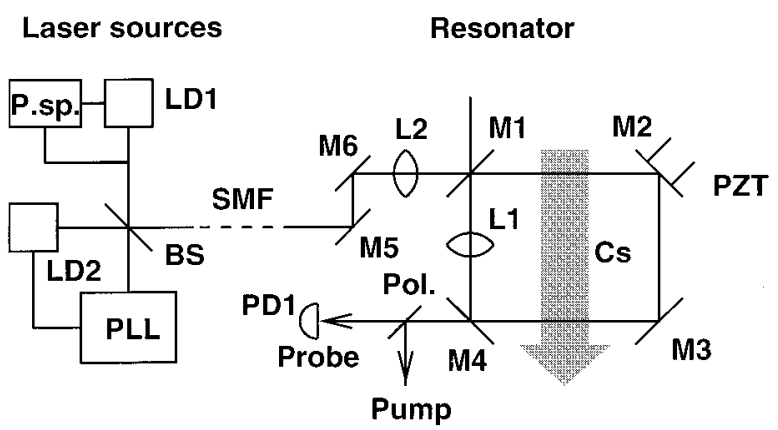

FIG. 4. Experimental setup. LD1, pump laser; LD2, probe laser; P.sp., polarization spectroscopy; PLL, phase locked loop; BS, power beam splitter; SMF, single-mode fiber; M2, M3, M5, and M6, mirrors; M1 and M4, polarization beam splitters; L1 and L2, lenses; Pol., polarization beam splitter; PD1, photo diode; PZT, piezoelectric transducer; Cs, cesium atomic beam.

prestabilized by a self-injection locking setup (Hollberg setup) ([20] and references therein). The frequency of the pump laser (LD1) was stabilized with a polarization spectroscopy technique (P.sp.) on the $6 s_{1 / 2} F=4 \leftrightarrow 6 p_{3 / 2} F^{\prime}=4$ transition (e.g., [21]). The laser fields had orthogonal linear polarizations and were superimposed at a power beam splitter (BS). The first output of this beam splitter was used to detect the beat signal between the fields. This signal was used to phase lock the probe laser (LD2) to the pump laser (LD1) with a difference frequency of around $9.2 \mathrm{GHz}$. This difference frequency was generated by a tunable microwave synthesizer (for details see [16], principles [22]). To achieve good overlap of the transverse modes of the fields the second output of the beam splitter was focused into a single mode fiber (SMF). After the fiber the error in the perpendicularity between the linear polarizations of the fields was in the range of $1 \%$.

The resonator was built around the vacuum chamber of the atomic beam with two polarization beam splitters as the input and output mirrors (M1, M4) with a reflectivity of $97 \%$ for the probe field and $4 \%$ for the pump field and two additional mirrors (M2, M3), which are highly reflective for both fields. To tune the length of the resonator, one of these mirrors was mounted on a piezoelectric transducer (PZT). With the antireflection (AR)-coated lens (L1, $f=200 \mathrm{~mm}$ ) inside the resonator we had an almost confocal ring resonator with a round-trip length of $80 \mathrm{~cm}$. The round-trip losses due to the four AR-coated vacuum windows and the lens were in the range of 5\%. We used two additional mirrors (M5, M6) and a second lens (L2) for beam alignment and mode matching. The linewidth of this resonator without atomic beam was 7.5 $\mathrm{MHz}$ (HWHM) with a coupling efficiency of $30 \%$. The diameter of the beam in both interaction regions was about 3 $\mathrm{mm}$.

First we measured the linewidth of the resonator with the atomic beam inside for the special case where the resonance frequency of the empty resonator was identical to the twophoton resonance frequency. For this we first phase locked the probe laser on the pump laser with a difference frequency equal to the two-photon resonance. Then we tuned the resonator with the PZT so that it was resonant with the probe field. After this the frequency of the microwave synthesizer and therefore the frequency of the probe laser was tuned 5 

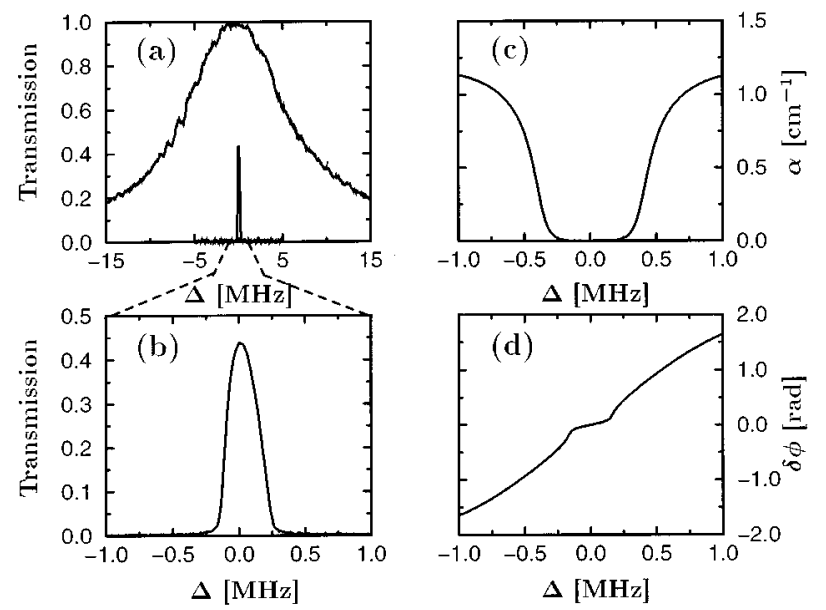

FIG. 5. (a) Measured transmission of the resonator without atomic beam (upper curve) and with. (b) Measured transmission with atomic beam. The linewidth without atomic beam was 7.5 $\mathrm{MHz}$ (HWHM) and with atomic beam $140 \mathrm{kHz}$ (HWHM). The intensities are $I_{\text {pump }}=220 \mu \mathrm{W}, I_{\text {probe }}=450 \mu \mathrm{W}$. (c) Calculated round-trip absorption $\alpha$ and (d) additional round-trip phase shift $\delta \phi$ ( $\propto$ dispersion) induced by the medium. Both curves are strongly bleached out by the power buildup of the probe field. The empty resonator is resonant at the two-photon resonance.

MHz below the two-photon resonance and scanned $10 \mathrm{MHz}$ over the resonance. The transmission of the resonator with and without atomic beam is shown in Fig. 5(a). The linewidth with atomic beam was $140 \mathrm{kHz}$ (HWHM) [Fig. 5(b)], which is 50 times smaller than the linewidth without atomic beam. The transmitted intensity is half of the transmitted intensity of the empty resonator. This was caused by additional losses on resonance due to background gas. Absorption in the background gas was mainly caused by the $6 s_{1 / 2}$ $F=3 \leftrightarrow 6 p_{3 / 2} F^{\prime}=2$ transition, which could not be coupled to the $6 s_{1 / 2} F=4$ level.

To compare the reduction of the linewidth with the reduction predicted from theory, we had to take into account saturation broadening, power buildup in the cavity, and the ratio between the interaction length and the length of the resonator. The power of the pump field in this scan was $220 \mu \mathrm{W}$ and the power of the probe field in front of the resonator 450 $\mu \mathrm{W}$. Due to the coupling efficiency of $30 \%$ for the empty resonator and $15 \%$ for the resonator with atomic beam and the power buildup in the resonator, the power of the probe field in the resonator without atomic beam on resonance was in the range of $4.5 \mathrm{~mW}$ and with atomic beam $2.25 \mathrm{~mW}$. Consequently the effective Rabi frequency $\Omega_{R}$ is determined mainly by the probe field when on resonance and by the pump field when off resonance. This leads to a strong saturation of the dispersion and the absorption on resonance. Therefore the edges of the transmission profile [Fig. 5(b)] are steeper than the edges of a typical Airy function. The righthand-side curves in Fig. 5 show the calculated absorption (c) and the calculated additional round-trip phase shift (d) ( $\propto$ dispersion) for these parameters. In both curves the strong saturation is easily seen. For these curves we have calculated the resonator internal intensity of the probe field and its influence on the absorptive and dispersive properties of CPT. In our calculations we took into account the residual Doppler
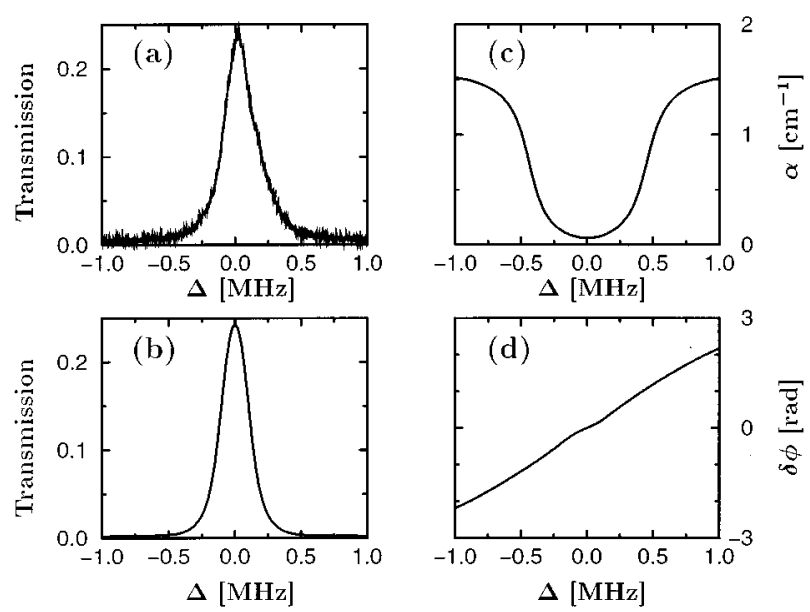

FIG. 6. (a) Measured transmission of the resonator with atomic beam. (b) Calculated transmission profile. The linewidth was again $140 \mathrm{kHz}$ (HWHM). (c) Calculated round-trip absorption $\alpha$ and (d) additional round-trip phase shift $\delta \phi(\propto$ dispersion) induced by the medium. With these intensities $\left(I_{\text {pump }}=300 \mu \mathrm{W}, I_{\text {probe }}=150 \mu \mathrm{W}\right)$ both curves are now not so strongly bleached out by the power buildup of the probe field. The empty resonator is resonant at the two-photon resonance.

broadening of the atomic beam, the power of the pump field, the power of the probe field including the buildup, the parameters of the resonator, and additional absorption due to the $6 s_{1 / 2} F=3 \leftrightarrow 6 p_{3 / 2} F^{\prime}=2$ transition, which depends only on the power of the probe field. We did not take into account the possible Zeeman splitting of the lower levels, which could be caused by imperfectly compensated magnetic fields and the Gaussian mode of the fields. We have assumed a constant intensity across the beam profile.

Next we increased the intensity of the pump field to 300 $\mu \mathrm{W}$ and reduced the intensity of the probe field to $150 \mu \mathrm{W}$. The linewidth for these parameters was again $140 \mathrm{kHz}$, but now the line profile was more similar to an Airy function (see Fig. 6). Curve (a) is a measured transmission profile, while curve (b) is calculated. The right-hand curves show the calculated absorption (c) and the additional round-trip phase shift (d) ( $\propto$ dispersion) induced by the medium for these parameters. The resonator internal power of the probe field on resonance was now about $450 \mu \mathrm{W}$, which is similar to the power of the pump field. Therefore the saturation of the dispersion and the absorption due to the power buildup is not so important. The transmitted intensity is now reduced to $30 \%$ of the transmitted intensity of the empty resonator. This can easily be explained by the higher absorption due to the background gas. This absorption rate only scales with the lower intensity of the probe field and cannot be saturated by the higher intensity of the pump field.

The line profile could be varied from a very sharp profile with steep edges by using more power for the probe field and less for the pump field to a profile which looks more like a smooth Airy function by using more power for the pump field and less power for the probe field. This behavior could easily be understood by taking into account the power buildup in the cavity and the saturation behavior of CPT.

So far we have only discussed how the transmission signal varies with the laser frequency in the case when the reso- 

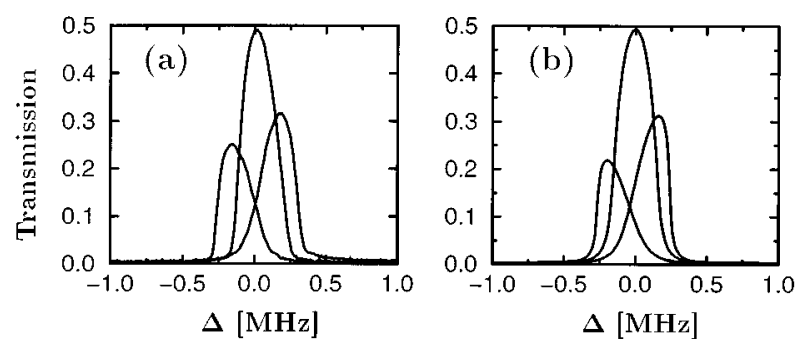

FIG. 7. (a) Measured transmission of the resonator red detuned, on resonance, and blue detuned relative to the two-photon resonance, (b) Calculated transmission profiles of the detuned resonator with a detuning of $0.29 \mathrm{rad}(17 \mathrm{MHz}), 0 \mathrm{rad},-0.45 \mathrm{rad}(-27$ $\mathrm{MHz}$ ).

nance frequency of the empty resonator is identical to the two-photon resonance. In this case the transmission signal is symmetric relative to the resonance frequency. For the experimental results presented in Fig. 7(a) the intensity of the pump field was again $220 \mu \mathrm{W}$ and of the probe field 450 $\mu \mathrm{W}$, as in the first experiment presented above (Fig. 5). The central line is again a profile which occurs when the resonance frequency of the resonator is equal to the two-photon resonance. For the left-hand curve the resonance frequency of the resonator is lower than the two-photon resonance frequency, for the right-hand curve it is higher. These curves are asymmetric with a steep edge at the outer side and a smooth intensity decrease at the inner side. At the steep edge the index of refraction of the medium was bleached out in the correct manner so that the detuning of the resonator could be compensated. In this frequency range the absorption is still high, because the difference frequency of the fields differs from the two-photon resonance. Therefore the amplitude of the transmitted signal decreases compared with the transmitted signal for a resonator tuned on the two-photon resonance. At the smooth edge we would expect the same behavior from the dispersion, but in addition the absorption decreases because now the difference frequency of both fields is in the range of the two-photon resonance. Both effects lead to this asymmetric line profile. In Fig. 7(b) the results from the numerical simulations are shown. For these simulations we introduced an additional round-trip phase shift as it would occur when the resonator was detuned. The phase shift of the right-hand curve corresponds to a frequency shift of $17 \mathrm{MHz}$ and, for the left-hand curve, of -27 MHz. These shifts should be compared with a linewidth (HWHM) of the empty resonator of $7.5 \mathrm{MHz}$. The transmission of an empty resonator with this length for this frequency would be vanishingly small. The steep saturable dispersion of CPT reduces the influence of the geometrical length of the resonator on the resonance frequency.

To summarize, we studied the influence of CPT on the properties of an optical resonator. Due to the steep nonabsorptive dispersion at the two-photon resonance the linewidth of the transmission signal in frequency space was 50 times lower than the linewidth of the empty resonator. The transmission profile depends on the intensity ratio of pump and probe field. This behavior occurs because of the power buildup of the probe field and the saturation of CPT. In addition, we studied the transmission behavior for a detuned resonator, and have shown that the influence of the length of the resonator on its resonance frequency decreases dramatically.
[1] E. Arimondo, in Progress in Optics, edited by E. Wolf (Elsevier, Amsterdam, 1996), Vol. 35, pp. 257-354.

[2] P. R. Hemmer and M. G. Prentiss, J. Opt. Soc. Am. B 5, 1613 (1988).

[3] M. O. Scully, S. Y. Zhu, and H. Fearn, Z. Phys. D 22, 471 (1992).

[4] A. S. Zibrov et al., Phys. Rev. Lett. 75, 1499 (1995).

[5] G. G. Padmabandu et al., Phys. Rev. Lett. 76, 2053 (1996).

[6] M. Fleischhauer and M. O. Scully, Phys. Rev. Lett. 69, 1360 (1992); Phys. Rev. A 49, 1973 (1994).

[7] J. E. Thomas et al., Phys. Rev. Lett. 48, 867 (1982).

[8] W. J. Childs, M. S. Fred, and L. S. Goodman, Appl. Opt. 13, 2297 (1974).

[9] A. Wicht, K. Danzmann, M. Fleischhauer, M. Scully, G. Müller, and R.-H. Rinkleff, Opt. Commun. 134, 431 (1997).

[10] C. Bradaschia et al., Nucl. Instrum. Methods Phys. Res. A 289, 518 (1990).

[11] A. Abramovici et al., Science 256, 325 (1992).
[12] K. Danzmann et al., in Gravitational Wave Experiments, edited by E. Loccia, G. Pizella, and F. Ronga (World Scientific, Singapore, 1995), pp. 100-111.

[13] K. Tsubono, in Gravitational Wave Experiments, (Ref. [12]), pp. 112-114.

[14] B. J. Meers, Phys. Rev. D 38, 2317 (1988); Phys. Lett. A 142 , 465 (1988).

[15] D. Schnier et al., Phys. Lett. A 225, 210 (1997).

[16] G. Müller, A. Wicht, R. Rinkleff, and K. Danzmann, Opt. Commun. 127, 37 (1996).

[17] P. M. Radmore and P. L. Knight, J. Phys. B 15, 561 (1982).

[18] M. Xiao et al., Phys. Rev. Lett. 74, 666 (1995).

[19] O. Schmidt et al., Phys. Rev. A 53, 666 (1996).

[20] C. E. Wieman and L. Hollberg, Rev. Sci. Instrum. 62, 1 (1991).

[21] W. Demtröder, Laser Spectroscopy (Springer-Verlag, Berlin, 1996).

[22] J. L. Hall, M. Long-Sheng, and G. Kramer, IEEE J. Quantum Electron. 23, 427 (1987). 